\title{
Aportaciones a la determinación de la prevalencia de la obesidad entre el alumnado de educación especial
}

\author{
F. Zurita Ortega ${ }^{a}$, R. Martínez Porcel ${ }^{b}$, OJ. Ali Morell, \\ R. Fernández García ${ }^{d}$, M. Fernández Sánchez ${ }^{\mathrm{d}}$ \\ aProfesor. Facultad de Ciencias de la Educación. Universidad de Granada. Granada. España. \\ ${ }^{b}$ Técnico especialista en psicomotricidad. Educador social. Fundación Purísima Concepción. Granada. España. \\ cFisioterapeuta. Fundación Purísima Concepción. Granada. España. \\ ¿Profesor. Facultad de Ciencias de la Salud. Universidad de Almería. Almería. España.
}

\section{Resumen}

La obesidad infantil está llegando a proporciones epidémicas en todo el mundo. La literatura sobre el nivel de obesidad de los niños con necesidades educativas especiales resulta escasa. Para abordar este problema, se revisaron las historias clínicas de 114 niños de 3 a 20 años del Colegio Purísima Concepción de Granada. Se analizaron datos demográficos, clínicos y antropométricos. El nivel de obesidad se determinó a partir del índice de masa corporal (IMC) y fue valorado según las curvas de percentiles para la edad y el sexo; según los resultados, los niños fueron clasificados como: peso bajo, normopeso, sobrepeso y obeso. Igualmente, los individuos fueron ordenados en seis grupos según el diagnóstico médico y psicológico emitido: síndrome de Down, autismo, parálisis cerebral infantil $(P C I)$, retraso mental, síndrome de baja incidencia y síndrome $X$ frágil. Se empleó el SPSS ${ }^{15.0}$ para el estudio estadístico.

De manera general se hallaron diferencias estadisticamente muy significativas en cuanto al sexo (mayor IMC en féminas) y la edad (aumento considerable coincidiendo con el incremento de la edad cronológica). Asimismo, se estableció una asociación por síndromes determinada por el 29,64 del IMC de los X frágil frente al 16,57 de la PCI. En cuanto a los niveles de obesidad, el $27,2 \%$ de la muestra tenía obesidad (determinada por los individuos con $X$ frágil y retraso mental), mientras que un $13,2 \%$ presentaba bajo peso, condicionado por factores de tipo social, metabólico y de desarrollo madurativo. Por ello, se harán necesarias la creación e implementación de programas que vayan orientados al aumento de la actividad física y de las mejoras nutritivas. sidad.

Palabras clave: Discapacidad. Educación especial. Índice de masa corporal. Nutrición. Obe-

Some contributions to the determination of obesity prevalence among children with special educational needs

Abstract

Obesity in children is reaching epidemic proportions throughout the world, with little literature on the level of obesity of children with special educational needs. To address this pro-

Félix Zurita Ortega, Felixzo@ugr.es, felixzo@hotmail.com

Los autores declaran no presentar conflictos de intereses en relación con la preparación y publicación de este artículo. 
blem, we reviewed the medical records of 114 children 3 to 20 years old of the Immaculate Conception School in Granada (Spain). We analyzed demographic data, clinical and anthropometric data. The level of obesity was determined from the tracking of BMI percentile curves by age and sex. The children were classified by the findings as underweight, normal weight, overweight and obese. Also the individuals were classified into six groups according to the medical diagnosis: Down Syndrome, Autism, Cerebral Palsy, Mental Retardation, Low Incidence Syndrome and Fragile-X Syndrome. SPSS ${ }^{1} 15.0$ was used for statistical studies. In general differences were statistically highly significant $(p=0.00$ ) with respect to gender (higher BMI in females) and age (increase coincided with an increase in chronological age). Association was also established with syndromes $(p=0.00)$, as determined by a BMI of $29.64 X$-Fragile versus $16.57 \mathrm{Ce}$ rebral Palsy. Twenty-seven and two percent of the sample was obese (defined by the Fragile- $X$ syndrome and mental retardation), whereas $13.2 \%$ were underweight, conditioned by social factors, metabolic and developmental maturity. It will be necessary the creation and implementation of programs aimed at increasing physical activity and improving nutrition.

Key words: Disabilities. Special education. Body mass index. Nutrition. Obesity.

\section{Introducción}

La obesidad se ha convertido en una de las principales líneas de investigación a nivel mundial. Se define como aquella situación en la que existe un desequilibrio entre el gasto y la ingesta energéti$\mathrm{ca}$, en el que prevalece un balance positivo y que trae como resultado el incremento del peso a expensas del tejido graso. La Organización Mundial de la Salud cita que, a nivel global, existen unos 250 millones de personas obesas, es decir, un $7 \%$ de la población mundial. Es un gran problema de salud pública en países desarrollados por el impacto que tiene en la esperanza y calidad de vida de los sujetos afectos, y es considerada por muchos como la epidemia más grave de la sociedad occidental del siglo $X X{ }^{1}$. Además, en individuos con necesidades educativas especiales estas cifras se hallan incrementadas ${ }^{2,3}$; sin embargo, es importante destacar la escasa literatura que existe sobre el tema de la obesidad asociada a la discapacidad psíquica ${ }^{4}$.

La obesidad como fenómeno social es simplemente el resultado de un desequilibrio por un destacado aumento del ingreso calórico cotidiano (sobrealimentación) junto con un marcado descenso del gasto calórico por el sedentarismo, situación que se asocia con el incremento de la prevalencia de enfermedades crónicas (cardiovasculares y diabetes). Debe añadirse que la obesidad infantil se asocia a un aumento del riesgo cardiovascular, a hiperinsulinemia con menor tolerancia a la glucosa, a alteraciones en el perfil lipídico en sangre, a hipertensión arterial, a problemas ortopédicos y a consecuencias psicosociales (como baja autoestima, aislamiento so- 
cial, discriminación y patrones anormales de conducta, incluidos los trastornos de conducta alimentaria). Asimismo, hay que tener en cuenta que los malos hábitos alimentarios y la inactividad física, así como los síndromes de anorexia y bulimia nerviosa, están afectando de manera cada vez más temprana a la población infantil y juvenil, y suponen un impacto mayor en los alumnos con necesidades educativas especiales ${ }^{5}$.

La obesidad, que implica numerosos factores y riesgos, es clasificada según su causalidad en endógena (por alteraciones fisiológicas) y exógena (por alteraciones conductuales); esta última es la más frecuente y en ella se contabilizan hasta un $90 \%$ de los casos. Los estudios epidemiológicos sugieren que las causas principales de la obesidad están relacionadas con los cambios ambientales y los estilos de vida, y resultan mucho menos frecuentes que otras enfermedades que se asocian a la obesidad, como el hipotiroidismo, el déficit de la hormona del crecimiento, el síndrome de Cushing, el pseudohipoparatiroidismo, el síndrome de Turner, los hipogonadismos, las lesiones del sistema nervioso central o la ingesta de fármacos como corticoides o antihistamínicos. También influyen factores de tipo hereditario, actitudes familiares ante la obesidad, hábitos de ejer- cicio o el nivel socioeconómico y cultural. En este contexto se incluyen también síndromes genéticos, enfermedades endocrinológicas y trastornos psicológicos ${ }^{6,7}$.

La adolescencia es una etapa decisiva en el desarrollo humano por los múltiples cambios fisiológicos y psicológicos que en ella ocurren. Éstos, a su vez, condicionan tanto las necesidades nutricionales como los hábitos de alimentación, la actividad física y el comportamiento. Además, está demostrado que estos hábitos repercuten en el estado de salud en la vida adulta. En este sentido no debemos obviar la discriminación que sufren los individuos obesos, que desencadena en ellos actitudes antisociales, depresión, aislamiento e inactividad, que inducen a aumentar la ingesta de alimentos y perpetuar el cuadro. Si a todo lo anterior le añadimos el hecho de padecer algún tipo de retraso mental, esta situación puede empeorar.

Padecer algún trastorno de tipo neurológico o mental en la infancia no implica ser obeso; sin embargo, la presencia de alguno de estos problemas se acompaña en muchos casos de problemas psicomotores, que suelen ser decisivos en la aparición de la obesidad. Al disminuir la capacidad motora de los individuos, se originan limitaciones fun- 
cionales y sensoriales que afectan a la autonomía del sujeto haciéndole, en la mayoría de los casos, dependiente de otro individuo, por lo que su aspecto motor se encuentra asociado al del cuidador; también la sobreprotección de padres y familiares que limitan las posibilidades del propio individuo pueden ser causantes de una mayor tendencia hacia la obesidad.

El sistema educativo actual, y más específicamente la educación especial, contemplan una educación integral de la persona e intentan dotar a los alumnos de una autonomía suficiente para afrontar las actividades cotidianas. Por ello, también es necesario formarlos para que sean capaces de tomar decisiones que vayan a tener consecuencias sobre su salud y la de los demás. Así, la educación física actual contribuye a la formación del hábito del ejercicio sistemático y a la promoción efectiva de los niveles de salud.

Si nos centramos en la obesidad, los docentes deben diseñar y desarrollar planes de tratamiento comprensivo, que deben incluir la prevención de la obesidad mediante el manejo de la actividad física y de la alimentación, la modificación del comportamiento $y$, cuando sea necesario, la participación familiar, incluyendo en esta área la educación especial como dis- ciplina integradora de individuos. Es importante informar acerca de la alimentación en general y educar sobre hábitos alimenticios saludables en particular, así como promover y facilitar el desarrollo de ejercicio físico a distintos niveles de actividad. Por tanto, la educación física y las ciencias de la salud pueden y deben asumir en su currículo este reto educativo que la sociedad actual plantea, pues no basta solamente con el tratamiento de la obesidad a nivel individual ni con la educación para la salud en las escuelas.

Siendo uno de los puntos de partida implicar a las comunidades educativa y sanitaria en la realización de un trabajo conjunto, es preciso impulsar acciones que contribuyan a erradicar esta epidemia del siglo XXI, concienciando a todos los sectores sobre la influencia que tiene la sobrealimentación en sujetos con necesidades educativas especiales.

En el presente trabajo sobre la valoración de la obesidad nos planteamos los siguientes objetivos: establecer el índice de masa corporal (IMC) medio de la población de estudio, así como estudiar qué tipologías de discapacidad presentan mayores niveles de obesidad.

\section{Material y métodos}

Esta investigación fue elaborada con un diseño de carácter descriptivo trans- 
versal, donde la variable estuvo representada por la determinación del IMC. Se empleó el SSPS ${ }^{\circledast} 15.0$ para el análisis estadístico.

\section{Población y ámbito de estudio}

La selección de la muestra se llevó a cabo en la población escolar del Centro de Educación Especial Purísima Concepción de Granada cumpliendo las normas éticas del comité de investigación y de la Declaración de Helsinki de 1975. La selección de la muestra definitiva se realizó por muestreo atendiendo a la composición natural de los grupos en el centro solicitado; además de la conformidad en la participación en el estudio, no existió ningún otro criterio de inclusión ni exclusión. La totalidad de los grupos fueron reclutados teniendo en cuenta las siguientes variables:
- Número de sujetos.

- Grupos de edad: 3 a 20 años.

- Sexo: masculino y femenino.

La muestra final utilizada en el estudio fue de 114 sujetos de 3 a 20 años; un $50,9 \%$ eran varones $(n=58)$ y un $49,1 \%$, féminas $(n=56)$. La edad de los individuos de este estudio está representada en la tabla 1.

Los sujetos estudiados fueron agrupados en categorías según el diagnóstico del dictamen de escolarización emitido por los equipos de orientación educativa, siguiendo la clasificación empleada por científicos de reconocido prestigio en el ámbito de la psicología y la neurología pediátrica ${ }^{8,9}$.

La distribución de los sujetos por la tipología del síndrome o diagnóstico fue bastante heterogénea (figura 1). Se debe añadir que al existir determinados síndromes con una muestra muy escasa,

\begin{tabular}{lccccc}
\hline \multicolumn{7}{l}{ Tabla 1. Frecuencias y } & proporcionalidad de los rangos de edad \\
\hline Edad (años) & N & Porcentaje & Edad (años) & N & Porcentaje \\
\hline 3 & 4 & 3,5 & 12 & 7 & 6,1 \\
4 & 4 & 3,5 & 13 & 8 & 7,0 \\
\hline 5 & 4 & 3,5 & 14 & 18 & 15,8 \\
6 & 5 & 4,4 & 15 & 3 & 2,6 \\
\hline 7 & 4 & 3,5 & 16 & 13 & 11,4 \\
\hline 8 & 7 & 6,1 & 17 & 11 & 9,8 \\
9 & 5 & 4,4 & 18 & 3 & 2,6 \\
10 & 7 & 6,1 & 19 & 6 & 5,3 \\
11 & 4 & 3,5 & 20 & 1 & 0,9 \\
\hline
\end{tabular}




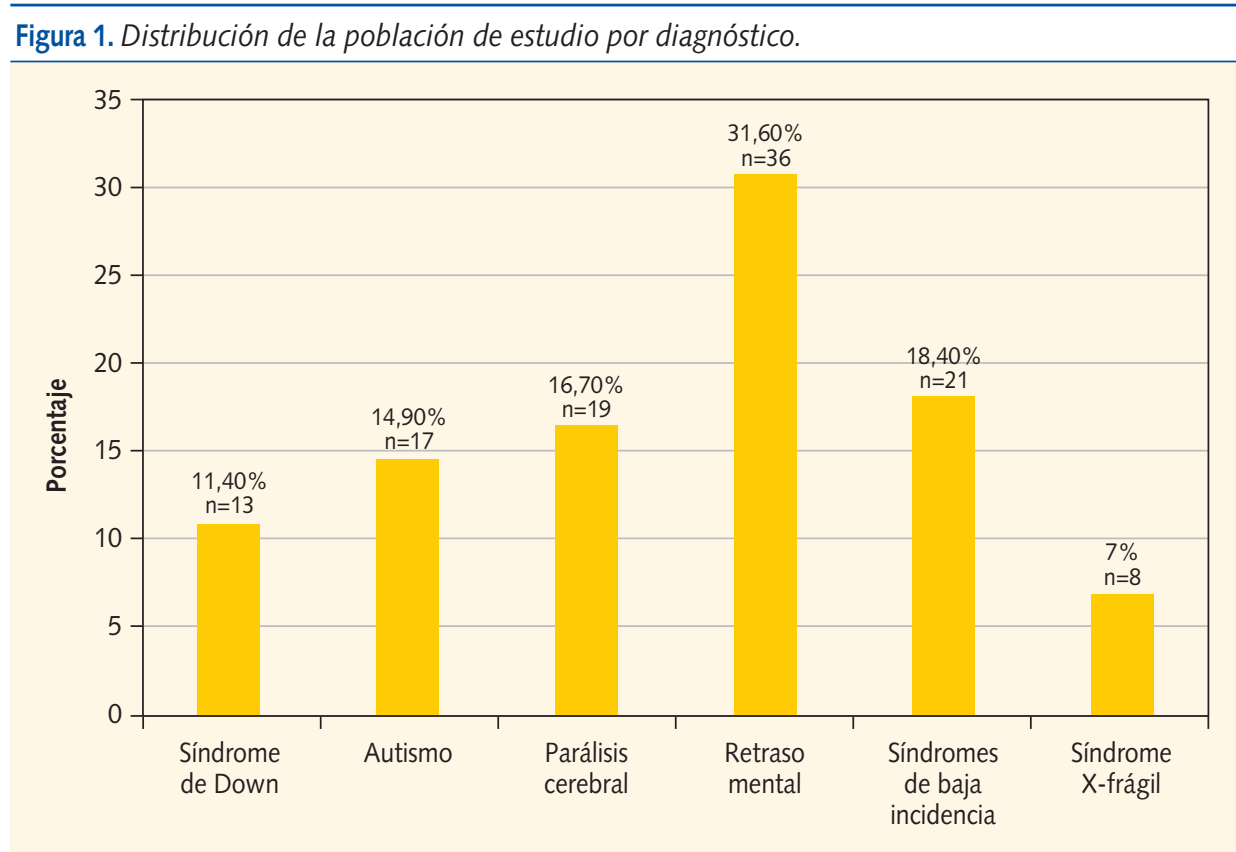

éstos fueron incluidos dentro de la categoría otras patologías (síndromes de Rett, Williams, Lennox-Gastaut, etc.).

\section{Variables}

La variable de nuestro estudio queda determinada por el IMC, para cuya realización se requiere:

- Medida del peso. Para la cuantificación de este parámetro, los sujetos estudiados se situaron con ropa ligera y descalzos en el centro de la báscula, sin acceso visual al registro de medida. El instrumento utilizado para la valoración del peso fue una balan- za (Seca MOD 881), con controlador digital, capacidad de un peso máximo de $200 \mathrm{~kg}$ y sensibilidad de $50 \mathrm{~g}$.

- Valoración de la talla de pie. La talla de pie es la distancia entre el vértex y el plano de sustentación del sujeto en estudio (plantas de los pies) medida en centímetros. Se instruye al estudiado para que mantenga la mirada al frente y realice una inspiración profunda en el momento de la lectura; el antropometrista le ayudará con una leve tracción hacia arriba desde el maxilar inferior. El sujeto perma- 
necerá en todo momento de pie guardando la posición de atención antropométrica con los talones, los glúteos, la espalda y la región occipital en contacto con el plano vertical del antropómetro; se emplea el Siber Hegner de cuatro secciones.

- IMC. Se calcula dividiendo el peso (en kilogramos) por la talla (en metros) al cuadrado. En el adulto, la obesidad se define a partir de un IMC superior a 30 , pero en los niños ésta tiene un carácter más relativo, por lo que hay que recurrir a las gráficas para cada edad y sexo $0^{10,11}$. Una vez que se establece el dato numérico obtenido de la fórmula, se realiza el encuadre en la categoría correspondiente, como se observa en la tabla 2.

\section{Resultados}

En este estudio de 114 sujetos, las féminas presentaron un IMC medio de 23,48 -desviación estándar $(D E)=9,17-$ frente al IMC medio de 20 de los varones $(D E=5,38)$. Se establecieron diferencias estadísticas muy significativas $(p=0,01)$ entre ambos grupos. El IMC medio de la muestra en general fue de 21,71. En cuanto a la edad, en la tabla 3 se observa cómo los sujetos presentaron una distribución muy heterogénea; aparecen 18

Tabla 2. Distribución numérica del índice de masa corporal en función del grado de obesidad para los distintos grupos de edad

\begin{tabular}{|c|c|c|c|c|c|c|c|c|}
\hline \multirow{2}{*}{$\begin{array}{l}\text { Edad } \\
\text { (años) }\end{array}$} & \multicolumn{2}{|c|}{ Bajo peso } & \multicolumn{2}{|c|}{ Normopeso } & \multicolumn{2}{|c|}{ Sobrepeso } & \multicolumn{2}{|c|}{ Obesidad } \\
\hline & Masculino & Femenino & Masculino & Femenino & Masculino & Femenino & Masculino & Femenino \\
\hline 3 & $<13,0$ & $<14,6$ & $13,0-19,0$ & $14,6-18,2$ & $19,1-21,3$ & $18,3-19,8$ & $>21,3$ & $>19,8$ \\
\hline 4 & $<13,4$ & $<13,9$ & $13,4-17,9$ & $13,9-18,7$ & $18,0-19,0$ & $18,8-22,8$ & $>19,0$ & $>22,8$ \\
\hline 5 & $<14,5$ & $<13,3$ & $14,5-17,9$ & $13,3-18,5$ & $18,0-21,3$ & $18,6-21,9$ & $>21,3$ & $>21,9$ \\
\hline 6 & $<14,4$ & $<13,6$ & $14,4-19,7$ & $13,6-19,4$ & $19,8-24,4$ & $19,5-22,8$ & $>24,4$ & $>22,8$ \\
\hline 7 & $<13,7$ & $<14,8$ & $13,7-19,2$ & $14,8-19,5$ & $19,3-24,6$ & $19,6-21,7$ & $>24,6$ & $>21,7$ \\
\hline 8 & $<14,1$ & $<14,3$ & $14,1-22,0$ & $14,3-20,6$ & $21,9-22,8$ & $20,7-21,4$ & $>22,8$ & $>21,4$ \\
\hline 9 & $<15,1$ & $<14,1$ & $15,1-21,9$ & $14,1-21,3$ & $22,0-23,2$ & $21,4-25,2$ & $>23,2$ & $>25,2$ \\
\hline 10 & $<14,8$ & $<14,5$ & $14,8-20,4$ & $14,5-21,9$ & $20,5-23,7$ & $22,0-25,0$ & $>23,7$ & $>25,0$ \\
\hline 11 & $<15,4$ & $<15,1$ & $15,4-22,7$ & $15,1-21,8$ & $22,8-23,7$ & $21,9-24,6$ & $>23,7$ & $>24,6$ \\
\hline 12 & $<16,3$ & $<15,5$ & $16,3-26,2$ & $15,5-22,1$ & $26,3-28,0$ & $22,2-24,7$ & $>28,0$ & $>24,7$ \\
\hline 13 & $<16,4$ & $<16,6$ & $16,4-24,8$ & $16,6-23,5$ & $24,9-27,2$ & $23,6-26,9$ & $>27,2$ & $>26,9$ \\
\hline 14 & $<16,8$ & $<17,2$ & $16,8-28,1$ & $17,2-23,8$ & $28,2-30,4$ & $23,9-27,4$ & $>30,4$ & $>27,4$ \\
\hline 15 & $<16,7$ & $<18,3$ & $16,7-26,0$ & $18,3-22,3$ & $26,1-30,4$ & $22,4-25,3$ & $>30,4$ & $>25,3$ \\
\hline 16 & $<17,7$ & $<17,7$ & $17,7-26,8$ & $17,7-23,9$ & $26,9-31,1$ & $24,0-27,8$ & $>31,1$ & $>27,8$ \\
\hline 17 & $<18,1$ & $<18,2$ & $18,1-25,6$ & $18,2-24,6$ & $25,7-28,3$ & $24,7-28,9$ & $>28,3$ & $>28,9$ \\
\hline$>18$ & $<18,1$ & $<18,0$ & $18,1-25,1$ & $18,0-25,7$ & $25,2-30,1$ & $25,8-28,3$ & $>30,1$ & $>28,3$ \\
\hline
\end{tabular}


Tabla 3. Distribución del índice de masa corporal medio en función de la edad

\begin{tabular}{lllllllc}
\hline Edad (años) & Media & N & DE & Edad & Media & N & DE \\
\hline 3 & 15,649 & 4 & 1,9349 & 12 & 18,941 & 7 & 3,9649 \\
\hline 4 & 13,602 & 4 & 1,1286 & 13 & 26,014 & 8 & 11,1704 \\
\hline 5 & 15,020 & 4 & 0,6241 & 14 & 23,751 & 18 & 6,0231 \\
\hline 6 & 13,958 & 5 & 1,9006 & 15 & 32,661 & 3 & 5,9010 \\
\hline 7 & 18,751 & 4 & 1,3724 & 16 & 25,153 & 13 & 5,8360 \\
\hline 8 & 15,434 & 7 & 2,4568 & 17 & 26,713 & 11 & 10,3198 \\
\hline 9 & 15,747 & 5 & 1,8406 & 18 & 36,001 & 3 & 2,6362 \\
\hline 10 & 17,957 & 7 & 2,7323 & 19 & 22,165 & 6 & 3,2013 \\
\hline 11 & 23,909 & 4 & 6,0262 & 20 & 17,000 & 1 & 0,000 \\
\multicolumn{7}{l}{ Total: X=21,711; $\mathrm{N}=114 ; \mathrm{DE}=7,6603$} \\
\hline \multicolumn{7}{l}{ DE: desviación estándar. } \\
\hline
\end{tabular}

individuos con 14 años frente a un único individuo de 20 años. Se observan amplias diferencias por edades $(p=0,00)$. Así, el 13,60 de IMC medio a los 4 años contrasta con los 36,001 de los 18 años; a partir de los 12 años, el IMC supera ampliamente el 20 frente a los valores que oscilaban en torno al 15 de las edades más tempranas en cuanto a la media del IMC.

Figura 2. Índice de masa corporal medio en función del sexo.

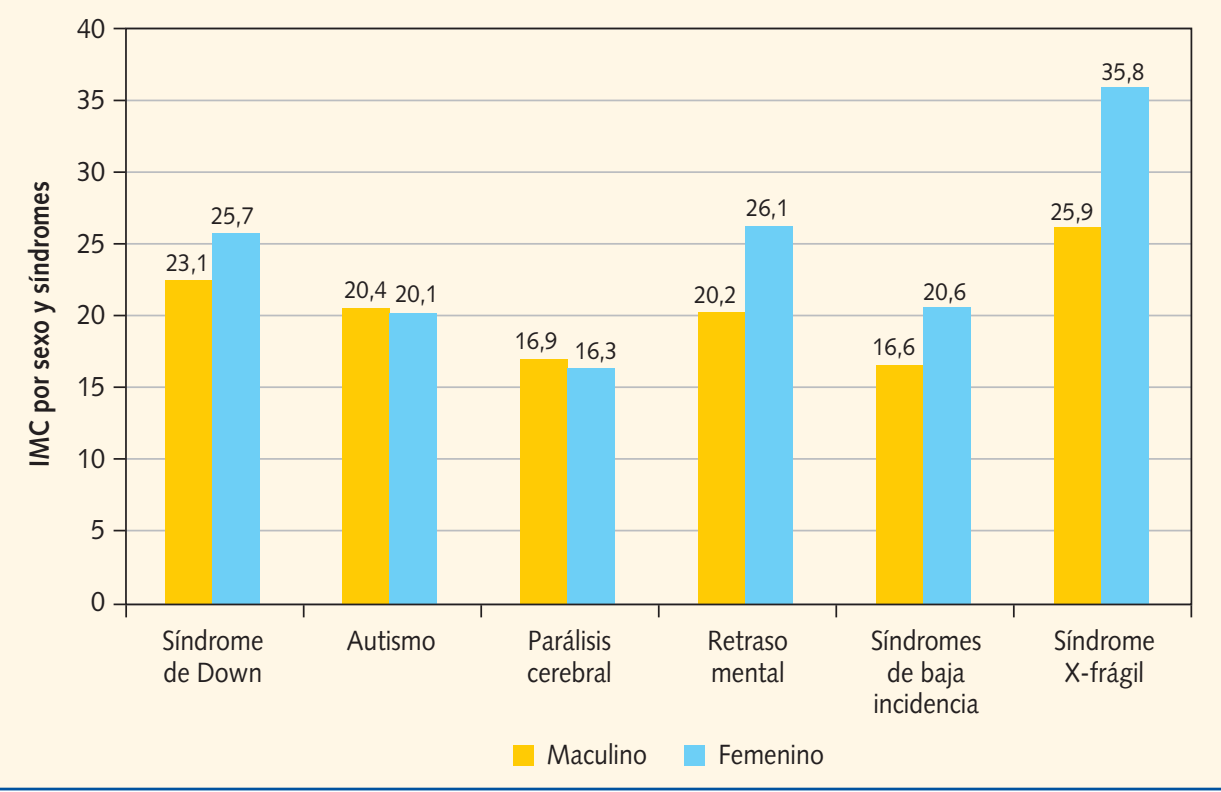


En la figura 2 exponemos el IMC medio de las diversas patologías o deficiencias objeto de nuestro estudio y los valores por sexo dentro de cada una de ellas.

Los mayores niveles de IMC los encontramos en los individuos con X-frágil $(X=29,64)$ frente a los sujetos con parálisis cerebral, que presentaron una media de 16,57 . Hay que señalar que entre los seis grupos definidos de personas con discapacidad mental apreciamos diferencias estadísticamente muy significativas $(p=0,00)$.

En cuanto al sexo, destacamos cómo en la parálisis cerebral infantil ( $\mathrm{PCl}$ ) y el autismo, los valores son similares entre niños y niñas; sin embargo, en el resto de categorías, las chicas obtuvieron un IMC más amplio que los varones y se produjeron diferencias estadísticamente muy significativas $(p=0,00)$, aspectos muy concordantes estadísticamente en lo referente a la edad, como mostramos en la figura 3.

En cuanto a los niveles de obesidad definidos en las cuatro categorías citadas anteriormente, 68 sujetos (59,6\%) tenían valores de normopeso y 15 $(13,2 \%)$ de bajo peso; el resto de la población $(27,2 \%)$ presentaba peso excesivo $(7,9 \%$ sobrepeso y $19,3 \%$ obesidad), como se muestra en la figura 4.

Figura 3. Índice de masa corporal medio según la edad y el tipo de enfermedad.

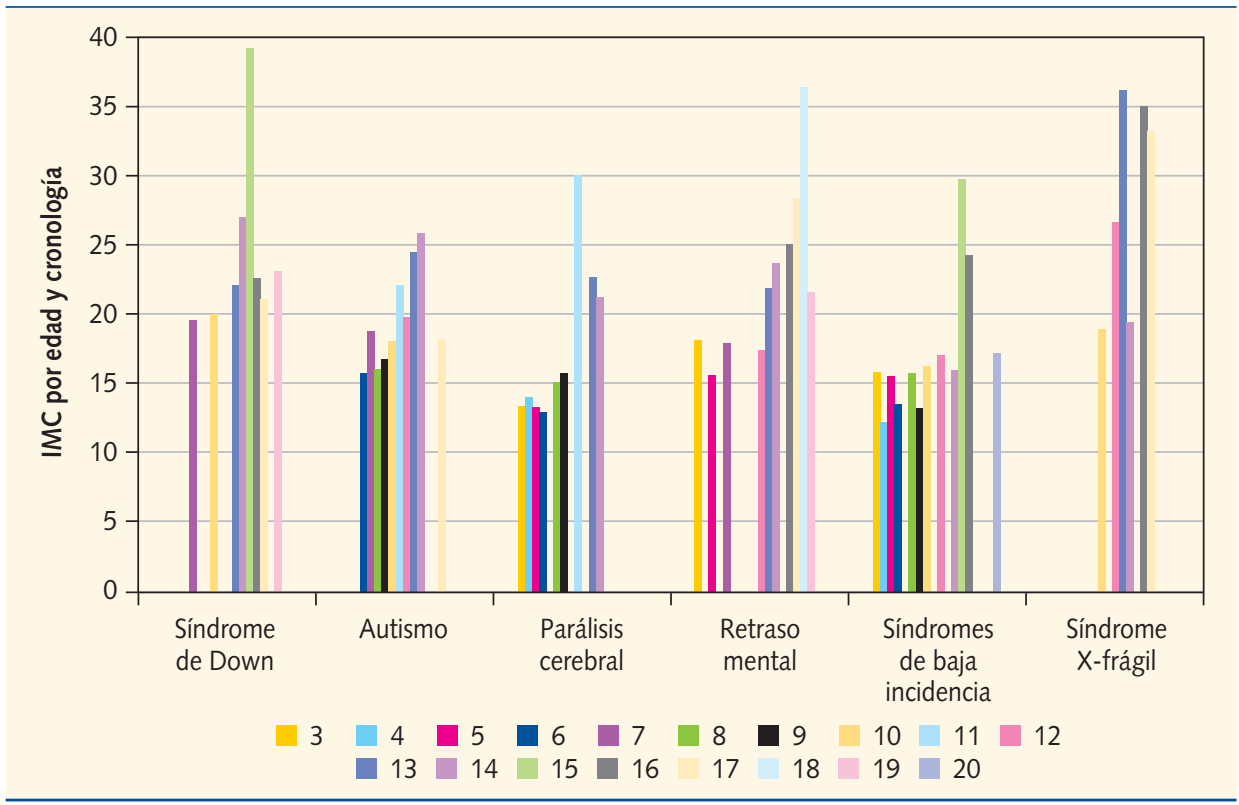


Figura 4. Distribución de las categorías de obesidad según el sexo.

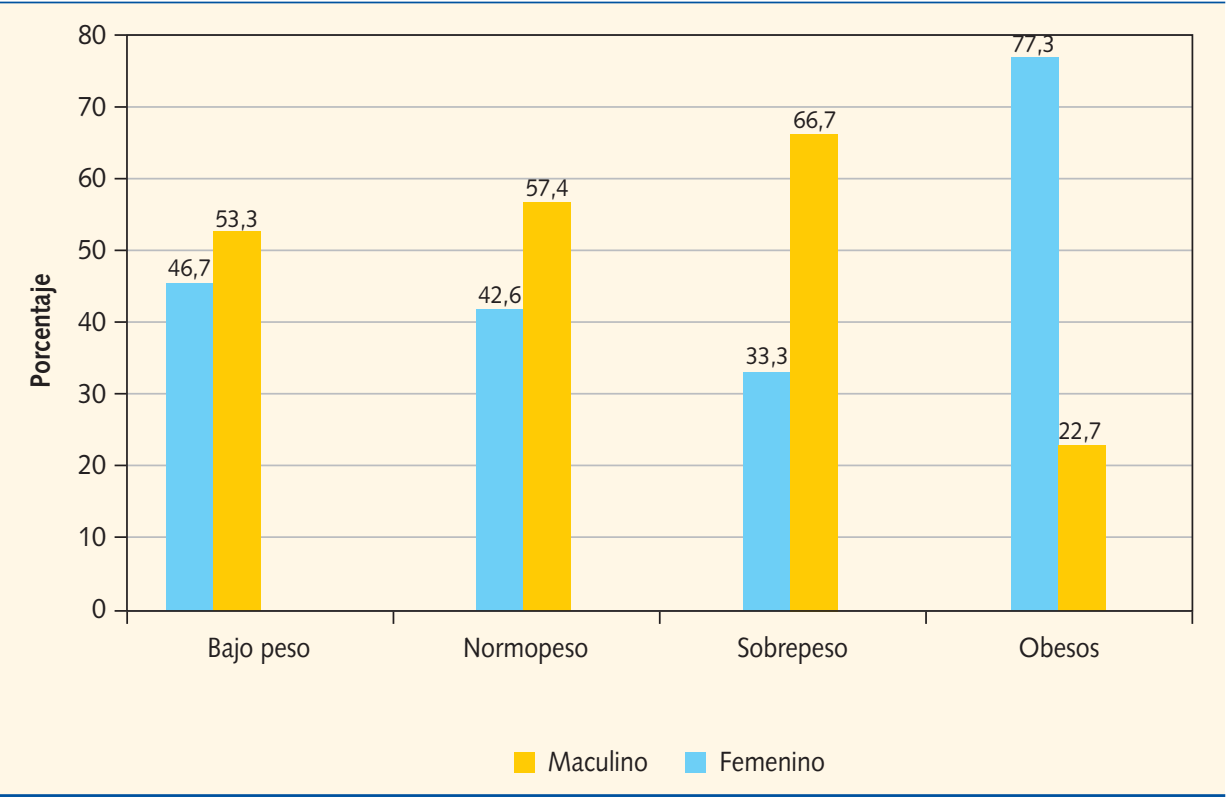

Figura 5. Obesidad por edad.

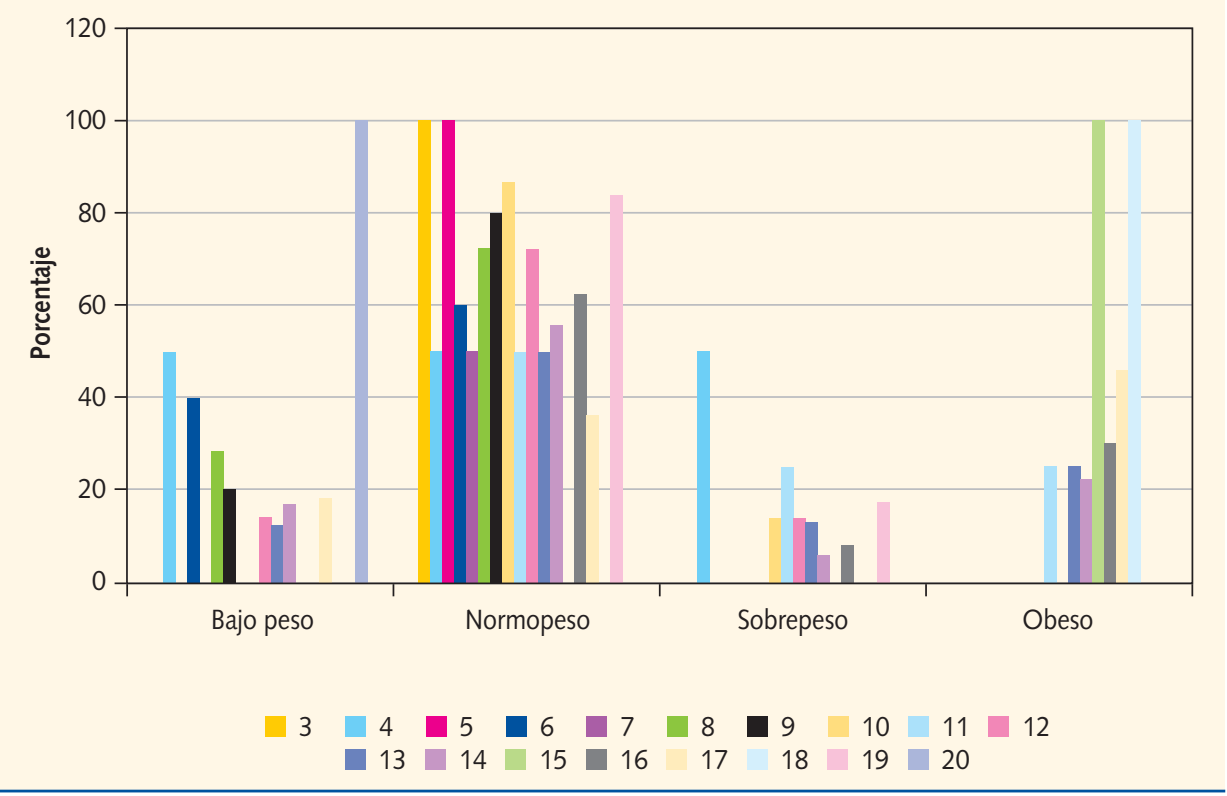


Entre los cuatro niveles de peso definidos se establecen diferencias $(p=$ 0,02 ) determinadas por la presencia de un mayor número de individuos con peso normal frente al resto de grupos. Por sexos destacamos que en bajo peso y en peso normal, los valores son prácticamente idénticos, difiriendo en sobrepeso $(66,7 \%$ de varones frente al $33,3 \%$ de mujeres) $y$, sobre todo, en obesidad, donde 17 (77,3\%) eran niñas frente a 5 chicos.

Asimismo, debemos observar cómo los sujetos varones con bajo peso superaban en cifra a los de sobrepeso y a los obesos $(13,8 \%$ frente a $10,3 \%$ y $8,6 \%$ respectivamente); en las féminas vemos que una tercera parte $(30,4 \%)$ eran obesas.

En lo referente a la edad, los resultados son muy heterogéneos en todas las categorías. Se obtuvieron valores estadísticamente muy significativos ( $p=$ 0,00 ), como se observa en la figura 5 . La obesidad se incrementó conforme avanzaba la edad cronológica.

En la tabla 4 y la figura 6 mostramos las cuatro categorías de IMC en función de los seis grupos descritos para personas con discapacidad en nuestro estudio; destaca la no aparición de diferencias significativas $(p=0,08)$. Así, del $13,2 \%$ de ca-

Figura 6. Diagnóstico por obesidad.

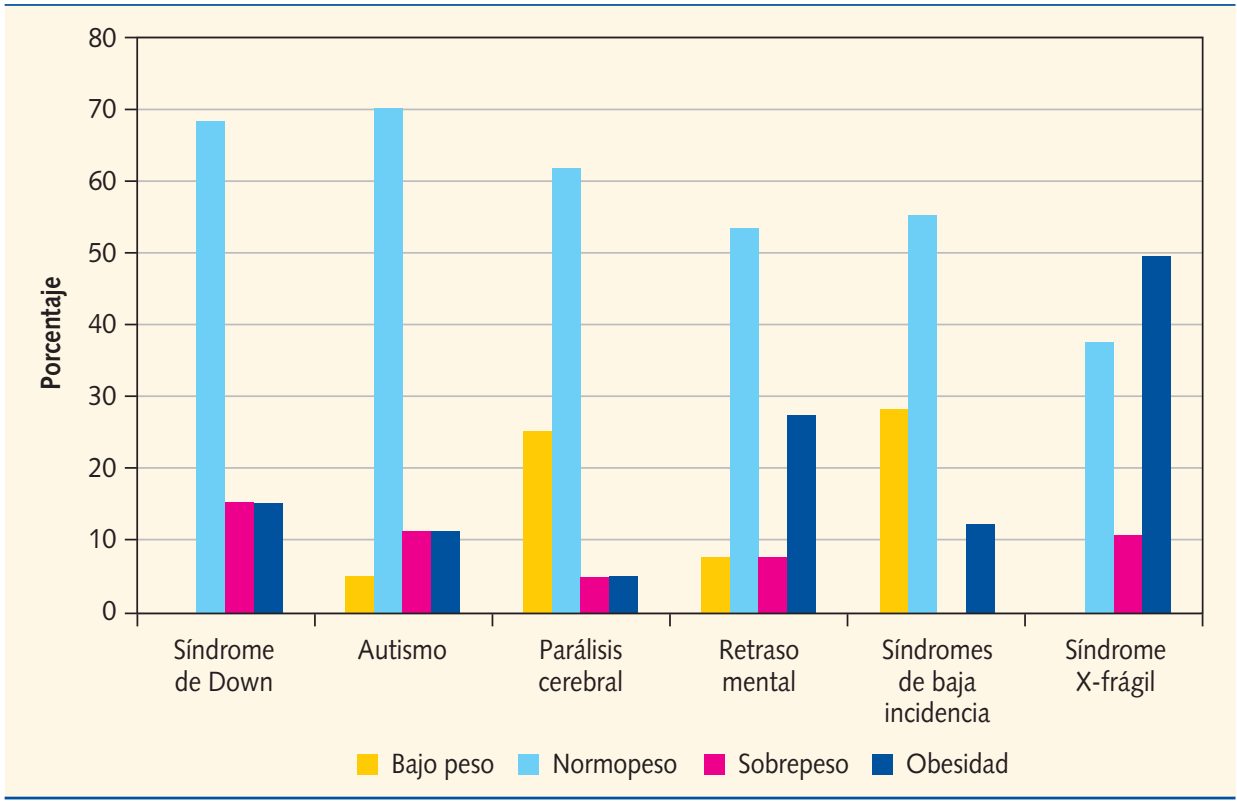




\begin{tabular}{|c|c|c|c|c|c|c|}
\hline Diagnóstico & & Bajo peso & Normopeso & Sobrepeso & Obeso & Total \\
\hline \multirow[t]{3}{*}{ Síndrome de Down } & $\mathrm{N}$ & 0 & 9 & 2 & 2 & 13 \\
\hline & Porcentaje de diagnóstico & 0,0 & 69,2 & 15,4 & 15,4 & 100,0 \\
\hline & Porcentaje de obesidad & 0,0 & 13,2 & 22,2 & 9,1 & 11,4 \\
\hline \multirow[t]{3}{*}{ Autismo } & $\mathrm{N}$ & 1 & 12 & 2 & 2 & 17 \\
\hline & Porcentaje de diagnóstico & 5,9 & 70,5 & 11,8 & 11,8 & 100,0 \\
\hline & Porcentaje de obesidad & 6,7 & 17,6 & 22,2 & 9,1 & 14,9 \\
\hline \multirow[t]{3}{*}{ Parálisis cerebral } & $\mathrm{N}$ & 5 & 12 & 1 & 1 & 19 \\
\hline & Porcentaje de diagnóstico & 26,3 & 63,1 & 5,3 & 5,3 & 100,0 \\
\hline & Porcentaje de obesidad & 33,3 & 17,6 & 11,1 & 4,5 & 16,7 \\
\hline \multirow[t]{3}{*}{ Retraso mental } & $\mathrm{N}$ & 3 & 20 & 3 & 10 & 36 \\
\hline & Porcentaje de diagnóstico & 8,3 & 55,6 & 8,3 & 27,8 & 100,0 \\
\hline & Porcentaje de obesidad & 20,0 & 29,4 & 33,3 & 45,5 & 31,6 \\
\hline \multirow{3}{*}{$\begin{array}{l}\text { Síndromes de baja } \\
\text { incidencia }\end{array}$} & $\mathrm{N}$ & 6 & 12 & 0 & 3 & 21 \\
\hline & Porcentaje de diagnóstico & 28,6 & 57,1 & 0,0 & 14,3 & 100,0 \\
\hline & Porcentaje de obesidad & 40,0 & 17,6 & 0,0 & 13,6 & 18,4 \\
\hline \multirow[t]{3}{*}{ SindromeX-frágil } & $\mathrm{N}$ & 0 & 3 & 1 & 4 & 8 \\
\hline & Porcentaje de diagnóstico & 0,0 & 37,5 & 12,5 & 50,0 & 100,0 \\
\hline & Porcentaje de obesidad & 0,0 & 4,4 & 11,1 & 18,2 & 7,0 \\
\hline \multirow[t]{3}{*}{ Total } & $\mathrm{N}$ & 15 & 68 & 9 & 22 & 114 \\
\hline & Porcentaje de diagnóstico & 13,2 & 59,6 & 7,9 & 19,3 & 100,0 \\
\hline & Porcentaje de obesidad & 100,0 & 100,0 & 100,0 & 100,0 & 100,0 \\
\hline
\end{tabular}

sos de bajo peso, los sujetos con el síndrome X-frágil y con síndrome de Down no aportan ningún individuo, y son los niños con síndromes de baja incidencia los que más casos presentan $(28,6 \%)$ junto con el $26,3 \%$ de sujetos con $\mathrm{PCl}$.

En cuanto a los sujetos con peso normal, los individuos con autismo, síndrome de Down y parálisis cerebral superan ampliamente el $63 \%$. El único que no llega al $50 \%$ es el síndrome $X$ frágil. El grupo de sobrepeso no alcanza cifras superiores al $15,4 \%$ en ningún nivel de discapacidad.
Por último, en lo referente a los obesos destacamos cómo el $50 \%$ de los $X$ frágil lo son; los siguen los sujetos con retraso mental $(27,8 \%)$. Por el contrario, solamente un niño con $\mathrm{PCl}$ se encuadró en este grupo (tabla 4).

Entre los individuos con síndrome de Down, no hay ningún sujeto de bajo peso, mientras que dos terceras partes de ellos presentan un peso normal. Valores muy similares en cuanto al peso normal se dan en el grupo de los autistas y en los niños con parálisis cerebral $(63,2 \%)$, aunque en este grupo una 
cuarta parte $(26,3 \%)$ son sujetos con bajo peso. Así, solamente el $55,6 \%$ ( $n=$ 20) de los individuos con retraso mental tiene peso normal, y el $27,8 \%$ de ellos son obesos, datos concordantes con el grupo con síndrome $X$-frágil, aunque éste obtiene mayores valores de obesi$\operatorname{dad}(50 \% ; n=4)$.

Mención aparte merecen los alumnos con otras patologías (síndromes de baja incidencia), con un $57,3 \%$ de normalidad y la presencia de un $28,6 \%$ de bajo peso. Hay que recordar que en este grupo se englobaban diversas patologías, por lo que debemos tratar con cierta precaución sus resultados.

\section{Discusión}

En el estudio realizado se da un IMC medio de 21,71 en los 114 individuos analizados, algo superior al hallado por Beauquis $^{12}$, en cuya población de estudio con rangos de edad y discapacidad similares al nuestro se obtuvo un 19,27, que contrasta con el 27,40 de IMC hallado por Melville et al..$^{13}$ en Inglaterra en sujetos con discapacidad intelectual. En nuestra población se establecen diferencias muy significativas por sexos ( $p=$ $0,01)$ condicionadas por el mayor IMC hallado en las niñas, con media de 23,48 , frente a una media de 20 en los niños, datos opuestos a los ofrecidos por otros autores ${ }^{14}$ que encontraron mayor incidencia en los chicos que en las chicas. Consideramos que este aumento viene originado por el inicio de desarrollo puberal más temprano en las niñas que en los niños, y que el comienzo de este desarrollo incluye un aumento corporal considerable. Asimismo, añadimos a esto las condiciones de índole social que rodean a este colectivo debido a su ubicación geográfica y que provocan un incremento considerable en los niveles de obesidad. Por el contrario, otros científicos ${ }^{15,16}$ no muestran en sus resultados, en cuanto a los niveles de peso, diferencias significativas por sexo, en la misma línea de lo expresado anteriormente. En cuanto a la edad, observamos un aumento gradual del IMC conforme avanza la edad cronológica, dato que sigue la evolución natural del IMC. De esta forma, entre los 3 y 10 años, el valor del IMC medio no supera la cifra de 17,95 frente al resto de edades, donde se incrementa de manera notable y llega incluso a 36, cifra alcanzada por 3 sujetos a los 18 años. Algunos autores $^{17-19}$ se manifiestan en este sentido cuando citan que durante los períodos de pubertad aumenta en un $40 \%$ la masa ósea, muscular y grasa, lo que coincide con lo observado en nuestra muestra, ya que se aprecia un patrón de aumento del IMC conforme se incrementa la edad 
de los escolares con discapacidad, tendencia similar a la de los individuos sin patología ${ }^{20}$.

Como se mostró anteriormente, la patología que presentó un mayor valor medio de IMC fue el síndrome X-frágil $(X=29,64)$, seguido del retraso mental y del síndrome de Down con valores mayores de 24 (superando estos tres la media de la muestra). En este senti$\mathrm{do}^{13,21,22}$, se expone que el síndrome $\mathrm{X}$ frágil se halla asociado a alteraciones de tipo endocrino y ocasiona altos valores de obesidad. Asimismo, destacamos cómo las tres chicas con este cuadro presentan valores de IMC dos veces superiores a sus homólogos. Del mismo modo, los individuos con retraso mental y síndrome de Down siguen la misma tendencia que los de otros autores consultados $^{12,23}$, y se afirma que el IMC es mayor en discapacitados de tipo cognitivo. Melville et al. ${ }^{13}$ refieren un IMC en adultos con síndrome de Down de 29,65 , lo que supone unas cifras muy elevadas en comparación con nuestro estudio. Consideramos que los controles nutricionales a los que se somete el alumnado de este centro pueden ser la causa de dicha disminución. Bruffaerts $^{23,24}$ afirma que los trastornos mentales tienen un efecto considerable sobre la incapacidad funcional, lo que da co- mo resultado una locomoción y una actividad de índole físico menores.

Del mismo modo, los niños con parálisis cerebral y síndromes de baja incidencia obtuvieron valores de IMC por debajo de 19, lo que confirma los datos referidos por otros autores ${ }^{23,25}$, y los autistas se mantuvieron en la media de la población; sin embargo, otros ${ }^{26}$ estiman que, debido a su patología, éstos siempre van a presentar índices globalmente menores que la población general.

En cuanto a la obesidad a nivel general, se establecieron diferencias estadísticamente muy significativas $(p=0,00)$. Así, el $13,2 \%$ de nuestra población obtuvo valores de bajo peso, dato que viene condicionado por el grupo de individuos con parálisis cerebral. Dicho conjunto se halla caracterizado por problemas de alimentación o trastornos nutricionales (de tipo oral, digestivo, etc.). Asimismo, casi el $60 \%$ de nuestra muestra se encuadró dentro de la normalidad, lo que recuerda la presencia tan heterogénea de población.

Debemos destacar que la suma de sujetos con sobrepeso y obesos nos dio un porcentaje del $27,2 \%$, valores muy parecidos a los hallados en poblaciones con sujetos totalmente sanos ${ }^{27-29}$; asimismo, en estudios similares al nuestro se observaron tasas de peso excesivo muy supe- 
riores a las nuestras ${ }^{4}$. Debemos considerar que en la revisión de la literatura existen escasos estudios como el nuestro.

En cuanto al sexo y la edad, destaca que las féminas presentan valores más altos de obesidad que los varones $(30,4 \%$ frente a $8,6 \%$ ), dato que, intuimos, viene determinado por las condiciones sociales de las cuales proviene la mayor parte de la muestra (zonas desfavorecidas), donde aún se considera un problema tener una niña con alteración cognitiva o funcional, y donde el varón tiene una mayor independencia a nivel motor. En cuanto a la edad, y recordando que se han seguido las curvas de crecimiento propuestas ${ }^{10}$, conforme se incrementa la edad cronológica se produce un aumento considerable de la obesidad ${ }^{18,20}$; asimismo, los individuos con parálisis cerebral son los que más bajo peso presentan (26,3\%), datos similares a los aportados por la mayor parte de los autores consultados ${ }^{30}$. Por último, destacar cómo, por síndromes, el Xfrágil presentó a la mitad de su población con obesidad (50\%). Hay que recordar cómo la obesidad está implícita en estas patologías y cómo algunos síndromes están en el $0 \%$ de bajo peso, aunque no presentan valores muy altos de obesidad, lo que viene determinado, en cierta medi$\mathrm{da}$, por el seguimiento a nivel general de pautas de tipo nutricional y motor (aspecto físico-deportivo).

\section{Conclusiones}

Los niveles de obesidad en nuestra población están por debajo de los de otros estudios similares. Se observa un incremento notable de la obesidad en el sexo femenino y un aumento de la proporción de obesos según avanza la edad cronológica.

La mitad de la población con síndrome $X$-frágil presenta valores de obesidad, mientras que los sujetos con parálisis cerebral tienen altos índices de normalidad y bajo peso.

El aspecto nutricional y la actividad física generalizada constituyen dos elementos valiosos para prevenir la obesidad infantil, así como para aumentar la calidad de vida en individuos con necesidades educativas especiales.

\section{Bibliografía}

1. Bousoño C. Sobrepeso y obesidad infantil. Aclarando dudas. VI Reunión Anual de la Sociedad
Asturiana de Pediatría de Atención Primaria 2007. Oviedo, España.

2. Sadaf I, O'Rahilly S. Monogenic obesity in humans. Annual Rev Med. 2005;56:443-58. 
3. Cardo ME, Servera M, Llobera J. Estimación de la prevalencia del trastorno por déficit de atención e hiperactividad en población normal de la isla de Mallorca. Rev Neurol. 2007;44:10-4.

4. Vélez J, Fitzpatrick A, Barbosa C, Díaz M. Nutritional status and obesity in children and young adults with disabilities in Punta Arenas, Patagonia, Chile. Int J Rehabilitation Res (Phil). 2008;31:30510.

5. Aranceta J, Pérez C, Ribas L, Serra L. Epidemiología y factores determinantes de la obesidad infantil y juvenil en España. Rev Pediatr Aten Primaria. 2005;7:13-20.

6. Sánchez JM, Eirís J, Otero JL, Pavón P, Castro M. Repercusión del retraso mental y de la parálisis cerebral asociada sobre la maduración ósea. Rev Neurol. 2002;34:236-43.

7. Camprubí C, Gabau E, Artigas J, Coll MD, Guitart M. Del diagnóstico clínico al diagnóstico genético de los síndromes de Prader-Willis y Angelman. Rev Neurol. 2006;42:61-7.

8. Verdugo MA. Autodeterminación y calidad de vida en los alumnos con necesidades educativas especiales. Siglo Cero. 2000;31:5-9.

9. Wehmeyer $M$, Schwartz $M$. Self-determination and positive adult outcomes: a follow-up study of youth with mental retardation or learning disabilities. Exceptional Children. 1997;63:24555.

10. SEEDO (Sociedad Española para el Estudio de la Obesidad). Consenso Español 1995 para la evaluación de la obesidad y para la realización de estudios epidemiológicos. Med Clin (Barc). 1996; 107:782-7.

11. Salas J, Rubio MA, Barbany M, Moreno B. Grupo Colaborativo de la SEEDO. Consenso SEEDO 2007 para la evaluación del sobrepeso y la obesidad y el establecimiento de criterios de intervención terapéutica. Med Clin (Barc). 2007;128: 184-96.
12. Beauquis A. Problemas nutricionales y factores asociados a los mismos en pacientes con discapacidad. XXI Jornadas Multidisciplinarias - XI Jornadas de Enfermería y I Jornadas de Residentes y Concurrentes, noviembre 2004. Argentina.

13. Melville CA, Cooper SA, McGrother CW, Thorp CF, Collacott R. La obesidad en los adultos con síndrome de Down: un estudio caso-control. J Intellectual Disability Res. 2005;49:125-33.

14. Pajuelo J, Amemiya I. El uso del índice de Quetelet en el diagnóstico nutricional en niños. An Fac Med (Perú). 1996;57:103-8.

15. Pisabarro R, Recalde A, Irrazábal E, Chaftar E. Primera encuesta nacional de sobrepeso y obesidad en niños uruguayos. Rev Med (Uruguay). 2002;18:244-50.

16. Zurita F. Screening de las alteraciones raquídeas (escoliosis e hipercifosis) en la población escolar de 8 a 12 años de Granada. Tesis Doctoral. Granada: Universidad de Granada; 2007.

17. Buckler HM, McLachlan RI, McLachlan VB. Serum inhibin levels in polycystic ovary syndrome: Basal levels and response to luteinizing hormonereleasing hormone agonist and exogenous gonadotropin administration. J Clin Endocrinol Metab. 1988;66:798-803.

18. Qing $\mathrm{H}$, Karlberg J. BMI in childhood and its association with height gain, timing of puberty and final height. Pediatr Res. 2001;49:244-51.

19. Pastor P, Makuk DM, Reuben C, Xia H. Chartbook on trends in the health of American. Health, United States. Hyattsville, MD: Nacional Center for Health Statistics; 2002.

20. Garagorri JM. Hipercrecimientos: sistema diagnóstico. An Esp Pediatr (Barc). 2004;60:291-5.

21. Artigas J, Brun C. Tratamiento médico del síndrome X frágil. Rev Neurol. 2001;33:41-50.

22. Ferrando L. Trastorno por déficit de atención e hiperactividad: factores etiológicos y endofenotipos. Rev Neurol. 2006;42:9-11. 
23. Bruffaerts R, Demyttenaere K, Vilagut $G$, Martínez M. The Relation Between Body Mass Index, Mental Health, and Functional Disability: A European Population Perspective. Can J Psychiatry. 2008;53:670-89.

24. Sánchez JM, Eirís J, Otero JL, Pavón P, Castro $M$. Influencia de los factores sociofamiliares sobre el estado nutricional en los niños con retraso mental. Rev Neurol. 2002;34:1001-9.

25. Stukat KG. Conductive education evaluated. Eur J Special Needs Education. 1995;10:154-61.

26. Marcos A, Varela P, Santacruz L, Morand G. Nutritional status and immunocompetence in eating disorders. Eur J Clin Nutr. 1993;47:78793.
27. Elcarte $R$, Villa-Elizaga I, Sada J, Gasco M, Oyarzabal M. Estudio de Navarra (PECNA). Prevalencia de hipertensión arterial, hiperlipidemia y obesidad en la población infanto-juvenil de Navarra. Asociación de estos factores de riesgo. Acta Pediatr Esp. 1993;38:428-36.

28. Moreno LA, Sarría A, Mur M, Lázaro A, Bueno M. Body fat distribution in children of families with familial combined hyperlipidemia. Nutr Metab Cardiovasc Dis. 1994;83:941-5.

29. Chueca M, Azcona M, Oyarzabal M. Obesidad Infantil. An Sistema Sanitario Navarra. 2002; 25(1):127-41.

30. Schwartz MW, Seeley RJ. The new biology of body weight regulation. J Am Diet Assoc. 1997; 97:54. 